Anat. Inst. d. Mediz. Fak., Univ. Okayama (Vorstand: Prof. M. SEKI).

\title{
Untersuchung der Veränderungen der gereizten Binde- gewebszellen unter dem Ansatz des Epithels am Zahn bei der Maus.
}

\author{
菓牙への上皮接着の下の刺戟された結織細胞の変化を \\ 二十日鼠㲹て観察。
}

\section{Hajime ONO 小野 一. \\ (Eingegangen am 29. Januar 1957.)}

Die Gingivalschleimhaut besteht aus dem Epithel und der Lamina propria und fehlt an lockerer Tela submucosa sowie an Drüsen. Das Epithel berührt die leblose Oberfläche des Zahnes, ohne daß es dort eine echte Verbindung bildet. Der Ansatz des Epithels am Zahn ist also besonders beim Menschen selbst durch eine von außen wirkende geringe mechanische Wirkung leicht ablöslich. Die in der Zahnfleischtasche zwischen dem Zahn und Zahnfleisch vorhandenen schädlichen Stoffe, Viren und Bakterien haben daher oft die Gelegenheit, leicht unter den Epithelansatz einzudringen. Vielleicht kommt aus diesem Grunde beim Menschen in der Nähe des Epithelansatzes eine mehr oder weniger bedeutende Zelleninfiltration zustande, worauf von vielen Forschern aufmerksam gemacht wurde. Vor kurzem haben z. B. OSOGOE (1951), ORBAN (1952) und ZACHINSKY (1954) an dem Zahnfleisch von gesunden Menschen und TAKATA (1955) an dem von Menschen sowie von einigen Säugetierarten eine mehr oder weniger bedeutende Infiltration von Lymphocyten, Plasmazellen, polymorphkernigen Leukocyten $u$. a. beobachtet.

Über das retikuläre Gewebe unter dem Epithel der Schleimhaut liegt eine nähere Untersuchung von SEKI $(1941,1953)$ an der Nasen- und Nasennebenhöhle des Menschen vor. Danach sollen im Normalzustand Retikulumzellen die Grundlage des subepithelialen Bindegewebes darstellen, und freie Zellen sollen zwischen den Retikulumzellen nur in geringer Zahl vorkommen. Die in der gereizten Nasenschleimhaut zahlreich auftretenden Histiocyten, monocytären Formen und grobgranulierten Leukocyten sollen zum größeren Teil aus den Retikulumzellen und Fibrocyten entstehen. Neuerdings untersuchte YASUDA (1957) die Veränderungen der Retikulumzellen und Fibrocyten in der von der Epitheloberfläche her durch Diphtherietoxin gereizten Schleimhaut der Kieferhöhle des Kaninchens und konnte bestätigen, daß aus den Retikulumzellen und Fibrocyten über Übergangsformen, 
wie Retikulohistiocyten bzw. Fibrohistiocyten, schließlich Histiocyten entstehen. Es scheint nun sehr wichtig zu sein, die durch die von der Zahnfleischtasche her eingedrungenen Reizstoffe hervorgerufenen Veränderungen der Bindegewebszellen unter dem Epithelansatz am Zahn zu untersuchen. YOKOMIZO (1954) machte beim Hund Versuche zur Erzeugung der Erscheinungen der Alveolarpyorrhoe und stellte fest, daß ohne Verletzung des Epithelansatzes eine Infektion nicht leicht entsteht. Dabei wurde aber der Entstehungsweise von infiltrierenden Zellen wenig Beachtung geschenkt. Die folgende Arbeit befaßt sich mit den experimentell hervorgerufenen Veränderungen, besonders mit den Umwandlungen der gereizten Bindegewebszellen, im Gewebe in der Nähe des Ansatzes des Epithels am Zahn bei der Maus.

\section{Material und Methode.}

Bei erwachsenen Mäusen wurden vier Arten von Reizung auf das Zahnfleisch an der lingualen Seite des Zahnes angesetzt. Erstens wurde das Zahnfleisch von dem Zahnhals des oberen Backenzahnes ein wenig abgelöst, und in die so eröffnete Tasche wurde Krotonöl zu 20\% enthaltendes Olivenöl mit Hilfe eines kleinen Wattenknöllchens 5 Minuten lang hineingetan. Zweitens machte man einen kleinen, kaum zum Bindegewebe reichenden Schnitt in den Epithelansatz an einem gleichnamigen Zahn und tat in den Schnitt 10\% ige alkoholische Krotonöllösung 10 Sekunden lang hinein. Es wurden drittens Diphtherietoxoid (von TAKEDA) und viertens Staphylokokkentoxoid (von Tokyo-Denken) nicht verdünnt unter den Epithelansatz an einem gleichnamigen Zahn in ganz geringer Menge eingespritzt. Das Einspriten wurde an ein und derselben Maus auf den beiden Seiten in einem Abstand von 18 Stunden vorgenommen, so daß das Tier 6 und 24 Stunden nach dem Einspritzen getötet werden konnte.

Nach Ablauf von bestimmten Zeiten nahm man den Kopf der Maus ab, schnitt die Wangen auf, um die Mundhöhle zu öffnen, und fixierte das Ganze in 10\%igem Formalin einige Tage lang. Man schnitt den Kopf saggital durch. Die oberen Backenzähne wurden mit dem umgebenden Gewebe ausgenommen und in 5\%iger Trichloressigsäurelösung entkalkt, um sie dann in Celloidin einzubetten. Die $15 \mu$ dicken Längsschnitte des Backenzahnes in der buccolingualen Richtung wurden nach der Eisenhämateinlackmethode ein wenig dunkler gefärbt als üblich. Diese auf die Angabe von v. MÖLLENDORFF zurückgehende Färbung stellt die Zellen bis ihre feinsten Ausläufer gut dar.

Da man das mit Formalin fixierte Material zum Zweck der Entkalkung mit starker Säure behandelte, wurde die feine Struktur des zarten Cytoplasmas weitgehend zerstört und nicht mehr deutlich zu sehen, zumal wenn der Gewebeschnitt zu dunkel gefärbt wurde. Die nun wie eine Sil. 
houette erscheinenden Zellarten der retikulofibrohistiocytären Gemeinschaft ließen sich trotzdem zumeist nicht schwer voneinander unterscheiden.

\section{Ergebnisse der Untersuchung.}

a) Bindegewebszellen im normalen Gewebe in der Nühe des Epithelansatzes auf der lingualen Seite des oberen ersten Backenzahnes.

Unter dem Epithelansatz an dem Zahn sind Bindegewebszellen, wie in Abb. 1 und 2 dargestellt sind, ziemlich dicht vorhanden. Die Zellen scheinen zu etwa drei Viertel dünne Fortsätze oder Ausläufer zu besitzen und nur zu etwa einem Viertel freie Zellen zu sein. In der stärkerer Vergrößerung in Abb. 2 konstatiert man, daß die größeren Zellen von länglicher Gestalt oder mit dünnen Ausläufern zu den Retikulumzellen oder Fibrocyten gehören. Die kleinsten rundlichen Zellen sind Lymphocyten. Große Histiocyten und sterbende Leukocyten werden mitunter angetroffen.

b) Veränderungen der Bindegewebszellen nach dem Hineintun von Olivenöl mit 20\%igem Krotonöl zwischen dem Zahn und der Gingiva.

Früher pinselte MUKOHATA (1941) Krotonöl auf die äußere Haut der Maus und sah nach 3 Stunden, wie zahlreiche freie Zellen aus den Fibrocyten unter der Haut entstehen. Nach dem 5minutigen Hineintun von Krotonöl enthaltendem Olivenöl in den Spaltraum zwischen dem Zahn und der Gingiva geschehen aber nach 6 Stunden keine so starke Veränderungen im Bindegewebes in der Nähe des Epithelansatzes. Aber durch nähere Untersuchung wird bestätigt, daß die Retikulumzellen und Fibrocyten unter dem Epithel sich abrunden und vermehren (Abb. 3). In den tieferen Teilen des Gewebes finden sich keine Veränderungen, und die meisten Bindegewebszellen bleiben immer noch typische Fibrocyten.

Nach dem Ablauf von 12 Stunden vermehren sich freie Zellen unter dem Epithel. Die in Abb. 4 gezeigten Zellen sind aber zumeist als die Übergänge von den Retikulumzellen in die Histiocyten, d. h. Retikulohistiocyten, oder als Übergänge von den Fibrocyten in die Histiocyten, d. h. Fibrohistiocyten, anzusehen.

Die Mannigfaltigkeit der Zellformen bekundet uns ihre amöboide Bewegung.

Nach 24 Stunden werden die Veränderungen bei weitem stärker (Abb. 5). Auffallend ist uns die Vermehrung von Histiocyten neben der von Retikulohistiocyten und Fibrohistiocyten. Die Vermehrung dieser Zellen erreicht in diesem Stadium das Maximum. Die Histiocyten enthalten oft große Vakuolen im Zelleib.

Nach 3 Tagen kehrt das Zellenbild beinahe zum normalen zurück (Abb. 6). Man sieht zwischen den Zellen aus dem Exsudat entstandene fibrilläre Produkte oder kollagene Fasern. Die Zellen haben keine großen Vakuolen mehr. 
c) Veränderungen der Bindegewebszellen nach dem Hineintun von 10\%. iger alkoholischer Krotonöllösung in die kaum zum Bindegewebe reichende Schnittwunde am Epithelansatz am Zahn.

6 Stunden nach dem Hineintum der Lösung vermehren sich schon Retikulo- und Fibrohistiocyten beträchtlich, die U̇bergänge von Retikulumzellen bzw. Fibrocyten in Histiocyten sind (Abb. 7). Auch kleine, als Monocyt aufzufassende Zellen werden vielerorts getroffen.

Nach 12 Stunden vermehren sich typische große Histiocyten, welche meistens in ihrem Cytoplasma große oder kleine Vakuolen enthalten (Abb. 8). Unter den Histiocyten und ihren Vorstufen treten 12 und 24 Stunden nach der Einwirkung der Krotonöllösung zahlreiche auf, von denen sich, wie YASUDA (1957) in der entzündeten Schleimhaut der Kieferhöhle des Kaninchens bemerkt hat, Stïckchen des Cytoplasmas mit oder ohne kleine Vakuolen abtrennen oder deren große Vakuolen nach außen platzen. Die Konturen solcher Zellen zeigen eine zerklüftete Oberfläche (Abb. 8 u. 9). Kleine kugelige Zellen sind Lymphocyten oder Monocyten.

Nach 3 Tagen vermehren sich, wie in Abb. 10 dargestellt ist, Fibrocyten und Fibrohistiocyten ohne große Vakuolen. Das Bild des Gewebes nähert sich dem Normalen. Zwischen den Zellen bemerkt man reichliche fibrilläre Gebilde.

d) Veränderungen der Bindegewebszellen nach dem Einspritzen kleinster Menge vom Diphtherie- bzw. Staphylokokkentoxoid in das Gewebe unter den Epithelansatz am Zahn.

Über die Wirkung von Diphtherietoxoid auf die Bindegewebszellen liegt die Arbeit von SHINDO (1953) vor, der bei der Katze nach der intravenösen Injektion die Zellen im Unterhautgewebe untersucht hat. In meiner Untersuchung wurde das Toxoid direkt in die Gingiva injiziert. In der Zeit 6 Stunden nach der Diphtherietoxoidinjektion ziehen die meisten Retikulumzellen und Fibrocyten ihre Fortsätze zurück und wandeln sich in Retikulo- bzw. Fibrohistiocyten (Abb. 11). Die Tatsache, daß kleine freie Zellen zahlreich vorkommen, deutet darauf hin, daß die Reizung von Toxoid auf die retikulofibrohistiocytäre Gemeinschaft stark ist.

Nach 24 Stunden machen Histiocyten und ihre Vorstufen den größten Prozentsatz aller Zellen unter dem Epithel aus (Abb. 12). Sie schließen oft große Vakuolen ein. Die Wirkung des Staphylokokkentoxid ist eine stärkere. Im Ablauf von 6 Stunden nach der Injektion treten zahlreiche kleine freie Zellen auf (Abb. 13). Die Mehrzahl von ihnen muß aus lokalen Retikulumzellen und Fibrocyten entstanden sein, denn alle Übergangsformen zwischen ihnen sind vorzufinden. Nur ein Teil der kleinen Zellen mag aus der Gefäßbahn ausgewandert sein.

Daß kleine freie Zellen an Ort und Stelle entstehen, ist noch besser im Bild nach 24stündiger Ablauf in Abb. 14 ersichtlich. Man findet auch 
Monocyten, welche sich aus Retikulumzellen und Fibrocyten oder daraus entstandenen freien Zellen entwickelt haben. In Abb. 14 finden sich keine ausgebildete große Histiocyten. Alles in allem läßt sich sagen, daß das Eiterung leicht verursachende Bakterientoxin in der retikulofibrohistiocytären Gemeinschaft freie Zellen von kleiner Form in größerer Zahl entstehen läßt.

\section{Zusammenfassung.}

Das Gewebe unter dem Ansatz des Epithels am Zahn der Maus wurde durch verschiedene Verfahren mit Krotonöl, Diphtherie- und Staphylokokkentoxoid gereizt, nach dem Ablauf von verschiedenen Zeiten fixiert und in Gewebsschnitte zerlegt und mit Eisenhämateinlack gefärbt. Da das Gewebestück zum Zweck der Entkalkung des Knochens mit Trichloressigsäure behandelt wurde, ging zwar die feine Struktur des Cytoplasmas weitgehend verloren, die Kontur der Zellen aber blieb noch deutlich, so daß die Arten der Bindegewebszellen sich ohne Schwierigkeit voneinder unterscheiden ließen.

1. In dem normalen Gewebe unter dem Epithelansatz am Zahn und in der benachbarten Gegend bilden die Retikulumzellen und Fibrocyten die Grundlage. Wenn toxische Stoffe auf diese Zellen wirken, können aus den Grundzellen verschiedene Zellformen entstehen. Nach dem Hineintun von in Olivenöl gelöstem Krotonöl in den künstlich gemachten seichten Spaltraum zwischen dem Zahn und dem Epithel werden die nämlichen Grundzellen nicht bedeutend verändert. Spritzt man aber eine sehr kleine Menge von Diphtherie- oder Staphylokokkentoxoid unmittelbar unter das Epithel ein, so entstehen aus den Grundzellen bakterienfeindliche Retikulound Fibrohistiocyten und aus diesen Histiocyten mit Vakuolen. Dazu kommen noch monocytäre Zellen von kleinerer Form. Nach dem Hineintum von alkoholischer Krotonöllösung in die Schnittwunde zwischen dem Zahn und dem Epithel geschehen etwa gleiche Veränderungen. Die Wirkung von Staphylokokkentoxoid bringt aber die stärksten Veränderungen hervor, indem es rasch zahlreiche kleine Zellen vorkommen läßt. Auf jeden Fall kehrt das Gewebsbild schon nach 3 Tagen beinahe zur normalen Gestalt zurück.

2. In den Stadien der starken und abklingenden Veränderungen des Gewebes treten in den Retikulohistiocyten, Fibrohistiocyten und Histiocyten Vakuolen auf. Häufig wird ein Teil des Cytoplasma mit oder ohne Vakuolen von ihrem Zelleib abgetrennt. Man sieht oft nach dem äußeren Medjum geöffnete Vakuolen. Die Zellen haben dann eine zerklüftete Oberfläche. 
H. ONO.

\section{内 容自抄。}

二十日鼠にては歯牙への上皮接着の附近の健常な結合組織には細網細胞 と線維細胞が基礎をなしている，歯牙と上皮の間を浅く開いてオリーブ油 そ溶かしたクロトン油を暫く大れるだけでも，結合組織の細胞に変化が起 るが，変化はその部分に切創を作ってアルコールに溶したクロトン油を10 秒間入れたり，またヂフテリートキソイドヌは葡萄球菌トキソイドを注射 したりすれば強く起る。即ち上記の基礎細胞から闘病に有力な, 云わば菌 敵細胞である網組球と線組球を経て組織球ができ，また刺戟が甚だ強い場 合には小形の単球様細胞が多く生ずる。変化の高期から恢復期にかけて網 組球，線組球，組織球の胞体と大きな空胞が現れる。それ等の細胞の胞体 の一部が小空胞を持ち又は持たないま〉外飞離れ，また大きな空胞が外界 そ開くのが屡ばであり，その細胞の表面は裂け目や割れ目を多く持つょう そなる。

\section{Literatur.}

Mukohata, J. : Experimentelle Untersuchungen über die Umwandlungsfähigkeit der Zellen im Subktangewebe der Maus. I. Okajimas Fol. 20 (1941). - Orban, B. : Histology and physiology of the gingiva. J. amer. dent. Ass. 44 (1952). - Osagoe, B.: Plasma cell infiltration in the gingiva. (Jap. m. engl. Zfass.) Kaibo Z. 26 (1951). - Takada, S.: A histological study of the round cellinfiltration in the gingiva. (Jap. m. engl. Zfass.) Kaibo Z. 30 (1955). - Seki, M.: Retikulumzellen und ihre Abkömmlinge in der Nasenschleimhaut des Menschen. Z. Zellforsch. 31 (1941); Arch. hist. jap. 4 (1953). - Shindo, T.: Veränderung des fibrohistiocytären und retikuloendothelialen Systems der Katze durch die intravenöse Injektion des Dyphtherietoxoides. Arch. hist. jap. 4 (1953). Yasuda, K. : Über die retikulofibrohistiocytäre Gemeinschaft der Kieferhöhlenschleimhaut des Kaninchens und ihre Antikörperproduktion, nebst einem Versuch über die Durchläßigkeit des Schleimhautepithels für Farbstoffe. Arch. hist. jap. 11 (1957). - Yokomizo, I. : Untersuchungen über die Entstehung der experimentellen Alveolarpyorrhoe. (Jap.) Shikai-tenbo. 11 (1954). — Zachinsky, L. : Range of histologic variation in clinically normal gingiva. J. dent. Res. 33 (1954). 
Tafelabbildungen. 


\section{Erläuterung $z u$ den Tafelabbildungen.}

Abb. 1-14. Aus dem Gewebe in der Nähe des Epithelansatzes an der lingualen Seite des oberen ersten Backenzahnes der Maus. Färbung der Schnitte mit Eisenhämateinlack.

Abb. 1. Normales Gewebe. $165 \times$ Ep Epithelansatz, * Schmelzsubstanz ist infolge der Entkalkung verloren.

Abb. 2. Vergrößerung des in der obigen Abbildung mit Linien umrahmten Abschnittes. $660 \times \quad F b c$ Fibrocyten, Lmc Lymphocyt, Rtz Retikulumzellen. 
II, ONO (1)

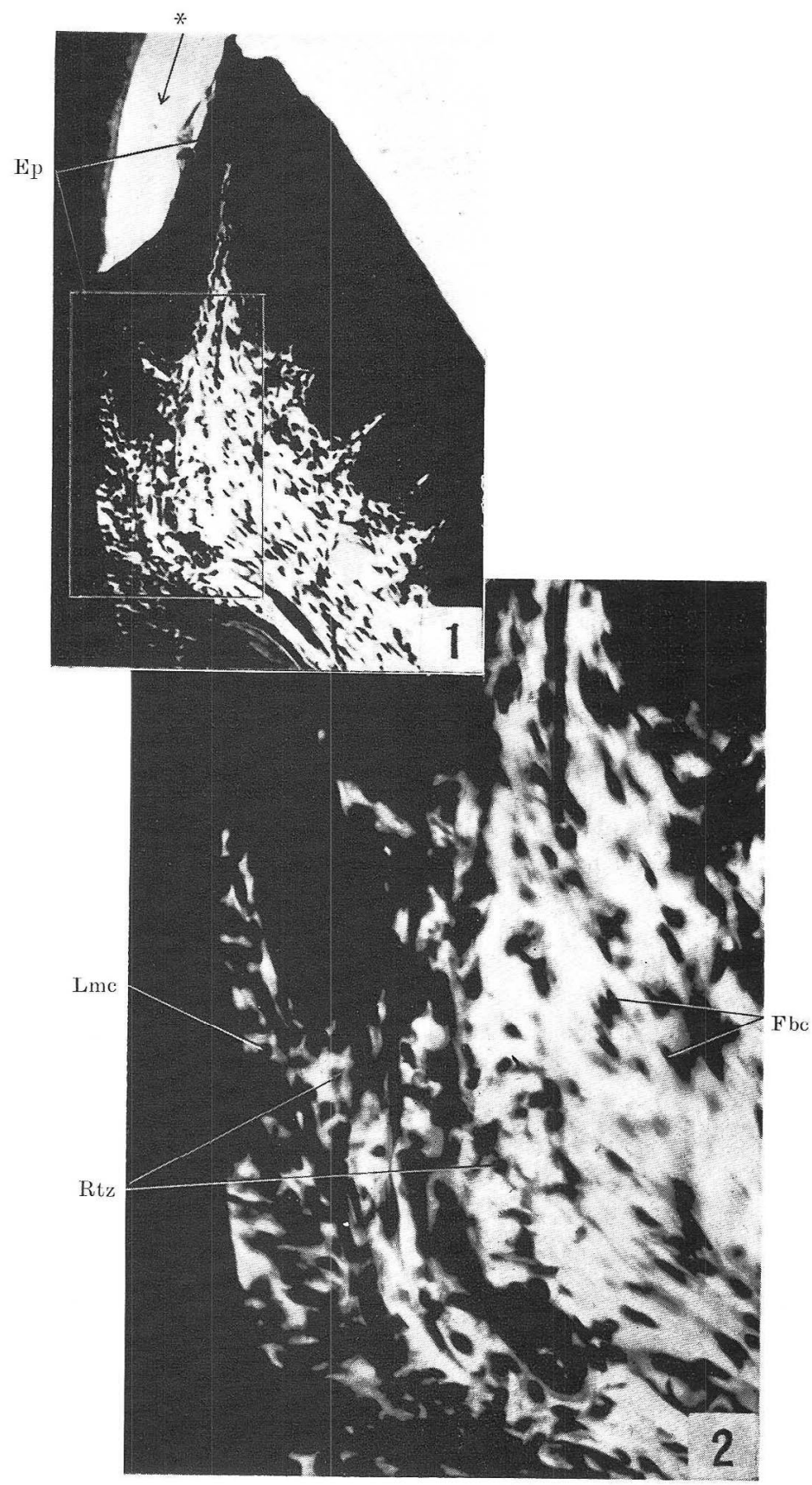

79 
Abb. 3. 6 Stunden nach dem Hineintun von Krotonöl-Olivenölgemisch zwischen dem Zahn und Epithel. $1100 \times \quad F b c$ Fibrocyt, * Abrundung und Vermehrung der Zellen.

Abb. 4. 12 Stunden nach dem Hineintun des Gemisches. $1100 \times$

Abb. 5. 24 Stunden nach dem Hineintun des Gemisches. $1100 \times H s c$ Histiocyten, Rthc Retikulohistiocyt, Rthc od. Fbhc Retikulo- oder Fibrohistiocyt, Rtz Retikulumzellen.

Abb. 6. 3 Tage nach dem Hineintun des Gemisches. $1100 \times$ 
II. ONO ( 2$)$
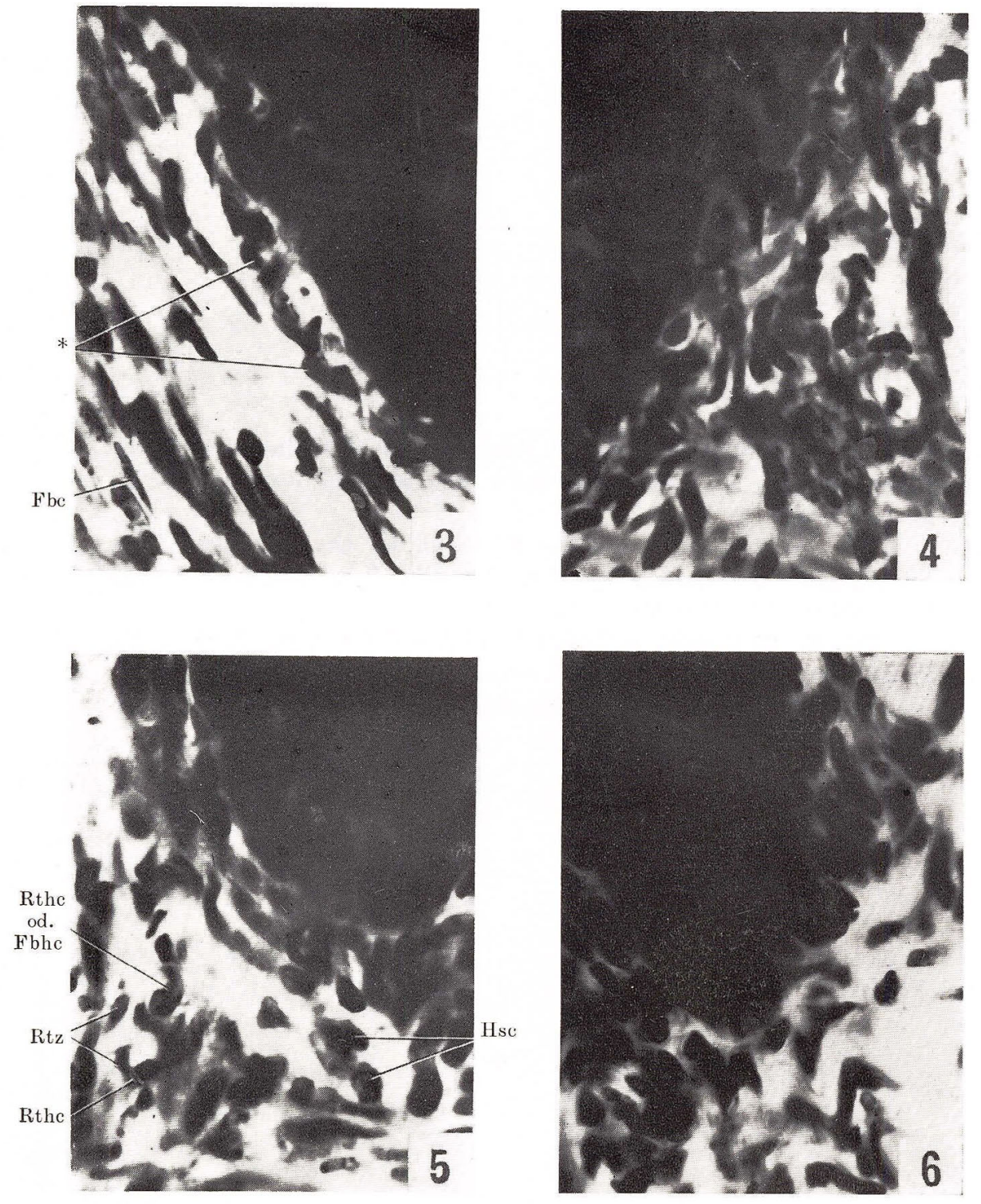
Abb. 7. 6 Stunden nach dem Hineintun von alkoholischer Krotonöllösung. $1100 \times M n c$ monocytoide Zelle, Rthc od. F'bhc Retikulo- oder Fibrohistiocyten.

Abb. 8. 12 Stunden nach dem Hineintun der Lösung. $1100 \times \quad F^{\prime} b c$ Fibrocyt, Hsc Histiocyten, Rtc Retikulumzelle, * Fortsatz des Retikulohistiocyten mit kleinen Vakuolen.

Abb. 9. 24 Stunden nach dem Hineintun der Lösung. $1100 \times H s c$ Histiocyten, Mnc Monocyt, Vak Vakuolen des Retikulohistiocyten.

Abb. 10. 3 Tage nach dem Hineintun der Lösung. $1100 \times \quad F b c$ Fibrocyten, Fbhc Fibrohistiocyten. 
H. ONO ( 3 )
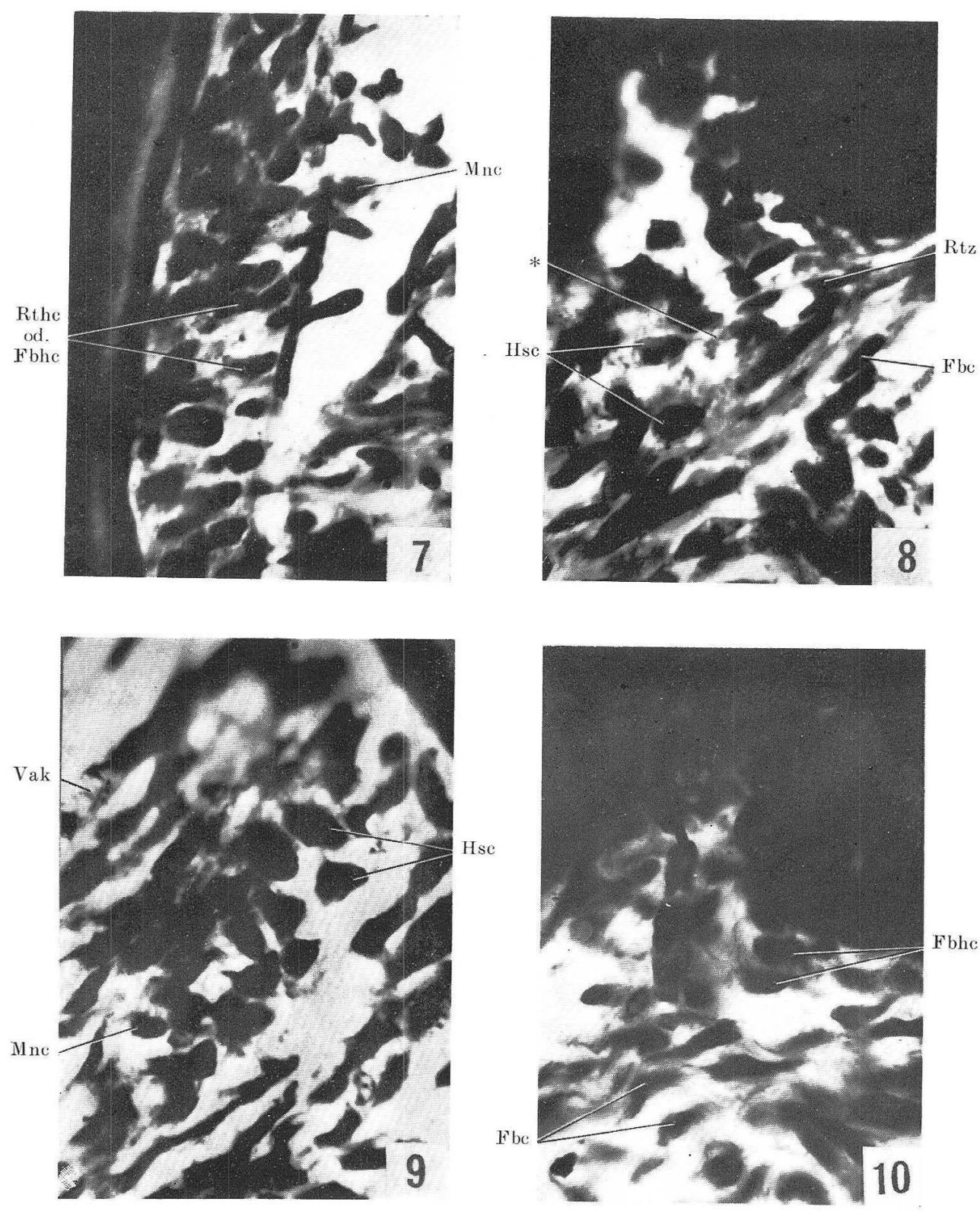
Abb. 11. 6 Stunden nach der: Injektion von Diphtherietoxoid. $1100 \times$ Rthc Retikulohistiocyt, Rtz Retikulumzellen.

Abb. 12. 24 Stunden nach der Injektion desselben. $1100 \times H s c$ Histiocyten, Rthc od. Fbhc. Retikulo- oder Fibrohistiocyten.

Abb. 13. 6 Stunden nach der Injektion von Staphylokokkentoxoid. $1100 \times *$ Übergänge zwischen Fibrocyten und kleinen freien Zellen, ** Übergänge zwischen Retikulumzellen und kleinen freien Zellen.

Abb. 14. 24 Stunden nach der Injektion desselben. $1100 \times$ Fhc Fibrocyt, Mnc monocytoide Zellen, Rtz Retikulumzellen. 
H. ONO (4)
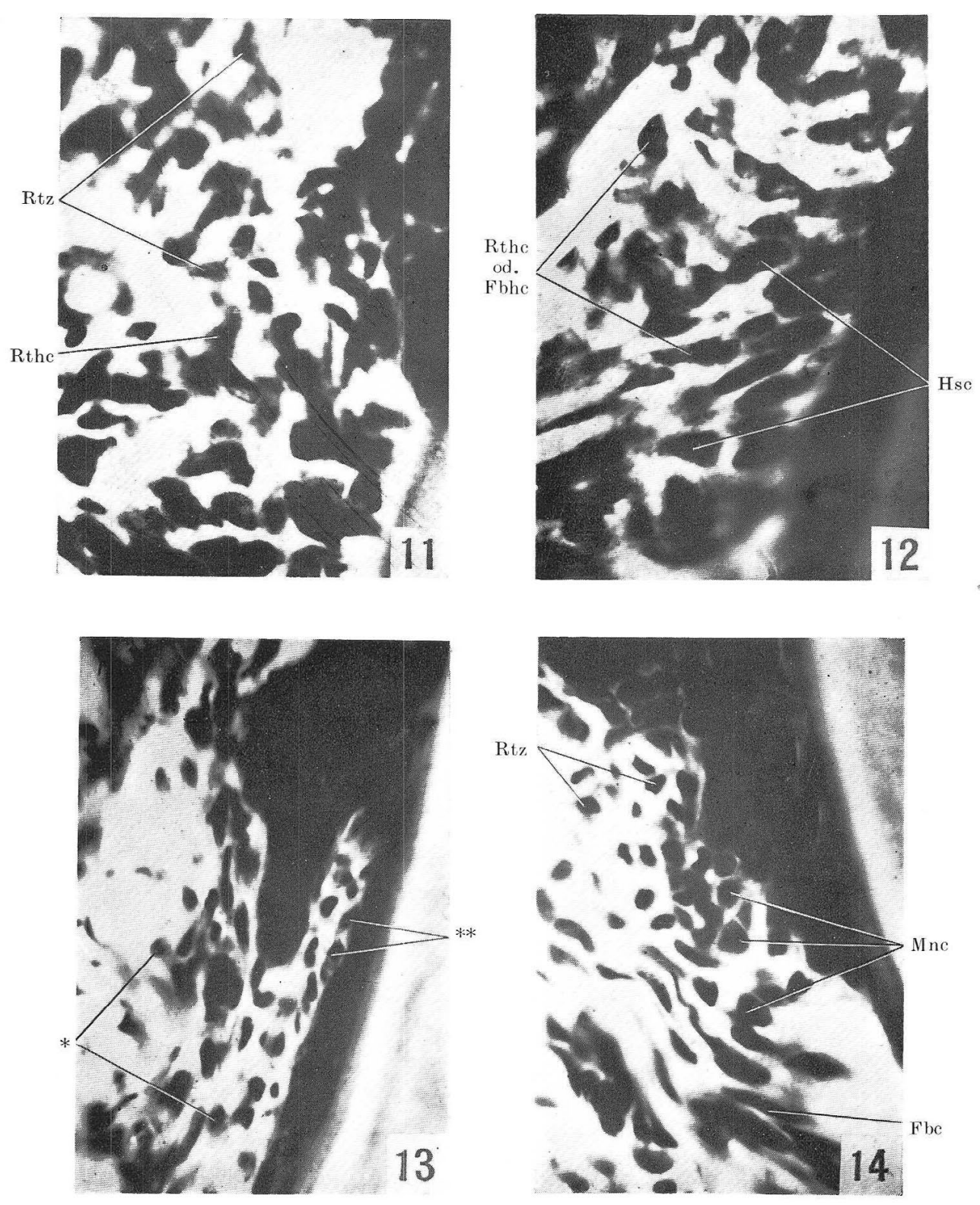